\title{
Results and Performance Analysis of an Efficient Reliable One Hop Broadcast in Mobile Adhoc Networks
}

\author{
Shinde Manisha K \\ PG Student, \\ MBES College of Engineering, \\ Ambajogai
}

\author{
V.R. Chirchi \\ Assistant Professor, \\ PG Dept, MBES COE, \\ Ambajogai
}

\begin{abstract}
Among Many fundamental communication primitives in mobile ad hoc networks, 1-hop broadcast plays an significant role in which a message from the source node is delivered to all nodes within the source node's transmission range. Although the importance of it, 1-hop broadcast is not easy to accomplish due to collisions in wireless networks known as Hidden Terminal problem. This paper presents a MAC protocol that guarantees 1-hop broadcast with efficiency by allowing as many simultaneous executions of the communication as possible without collisions. The proposed algorithm utilizes control packets that prevent data packet collisions, and at the same time, make the simultaneous communications possible to improve the network throughput.
\end{abstract}

\section{Keywords}

one hop broadcast, hidden terminal , manet,MAC protocol ,throughput ,packet ,efficient ,reliable broadcast ,node.

\section{INTRODUCTION}

A mobile ad hoc network is a collection of wireless mobile nodes that dynamically establishes the network in the absence of fixed infrastructure [1]. One of the characteristic features of MANET is, each node must be able to act as a router to find out the optimal path to forward a packet. As nodes may be mobile, entering and leaving the network, the topology of the network will change continuously. MANETs provide an emerging technology for civilian and military applications. Since the medium of the communication is wireless, only limited bandwidth is available. Another important restriction is energy due to the mobility of the nodes in nature. One of the important research areas in MANET is establishing and maintaining the ad hoc network through the use of routing protocols. Due to the nature of wireless networks, a transmission in the networks is basically a one-hop broadcast, in which a signal transmitted from a node (source node) reaches all nodes within its transmission range (neighbors of the source node). Many important algorithms in MANETs heavily depend on the performance of one-hop broadcasting.

Some significant applications of one-hop broadcasting are:

(1) Reactive routing algorithms in MANETs. In most reactive algorithms in MANETs [3], if the source node does not have route information to the destination node, the source node floods the network with a special control packet, Route Request Packet (REQ), to discover a path to the destination. This flooding process is based on onehop broadcast and continues until the REQ reaches the destination.

(2) Proactive routing algorithms that are based on Distance Vector Routing or Link State Routing. In implementing a proactive algorithm, each node maintains routing information in its routing table through periodic exchanges of its information with its neighbors. This exchange is usually done in the form of one-hop broadcast.

(3) Topology control. By exchanging necessary information with its neighbors, a wireless node adjusts its transmission range so that an efficient network topology is maintained for better energy conservation and network throughput [5].

(4) Algorithms based on hierarchical organization of nodes Especially, cluster-based algorithm needs one-hop broadcasting when the nodes are in the process of forming clusters. After a cluster is formed the head of the cluster must broadcast its election as a cluster head to all nodes in its cluster using one hop broadcast as well[2].

This paper is organized as follows: In section II, Literature Survey of paper. In section III, explained proposed method. In section IV, advantages proposed method. In section V, application and last section VI, conclusion.

\section{LITERATURE SURVEY}

Number of protocols and algorithms have been proposed in past for one hop broadcasting. Some are explain as follows.

A Stranded protocol IEEE 802.11 has been used in Wireless Local Area Networks (WLANs). IEEE 802.11 specifies Medium Access Control (MAC) for WLANs [3]. The performance of a wireless network depends upon the medium access control (MAC) protocol used. Carrier Sense Multiple Access (CSMA) protocol is often chosen because of its simplicity and scalability. However, CSMA is inclined to the hidden node problem [3], especially in ad hoc networks where a node may communicate directly with other node in range [1]. Hidden nodes cause packet collisions and thus considerably affect network performance. In order to conflict the hidden node problem, a mechanism known as RTS/CTS handshake is often used. The RTS/CTS mechanism was initially proposed in [1] in a protocol called Multiple Access with Collision Avoidance (MACA)[2].

It Avoids the network congestion resulting from frequent packet collisions. Can't explore simultaneous transmission. Work well in point to point communication. The False Blocking Problem and its Propagation .Park and Palasdeokar[8 ]have developed a reliable one hop broadcasting algorithm called ROB that utilizes only one channel for both control packets and data packets. ROB does not require any control packet transmission prior to data packet transmission. Broadcast Complete Problem that presents difficulty in detection in completion of one hop broadcast. Lembke et al. [9]proposed two reliable one-hop algorithms: one is proactive and the other is reactive. The reactive algorithm utilizes two channels and control packet called whistle which is similar to BIP in the proposed 
algorithm. Here Nodes cannot adjust their transmission ranges, Nodes do not explore as many simultaneous communication as possible to enhance the network throughput. The approach to dealing with node mobility is limited and not efficient. Whistle Propagation Problem, in which once a whistle is generated and propagated, it generates more whistles that live in the network forever causing the channels filled with useless whistles. Park and Anderson [8] have proposed a guaranteed one-hop broadcasting algorithm called GOB that resolves the Broadcast Complete Problem[7] and Whistle Propagation Problem[6]. GOB uses a control packet BIP to prevent possible collisions, and therefore, improve network throughput. It Prevents possible collisions ,Improve Network throughput. But Nodes do not explore simultaneous executions of one-hop broadcast.

\section{PROPOSED SYSTEM}

\subsection{Efficient Reliable one hop Broadcasting (EROB)}

EROB guarantees the completion of one hop Broadcasting i.e. all nodes in source's transmission range will receive the broadcast message.

The proposed System presents a significant improvement over the algorithms by exploring parallel executions of the 1hop broadcasting whenever possible, which produces far better performance in the network throughput.

EROB uses only a single type of control packet called Broadcast-In-Progress (BIP) to prevent collisions. A BIP is produced and used in two cases.

1) Prior to 1-hop broadcast of a data packet, a node transmits a BIP to secure not only the broadcast area but also broadcast threatening area to prevent any possible collision.

2) On receiving a BIP, a node that is currently involved in any other communication generates and transmits a BIP to warn other nodes in its broadcast threatening area not to initiate data packet transmission.

A BIP consists of unique bit pattern that distinguishes it from other types of packets. In addition to the actual data, a data packet contains other information such as the source's ID, packet ID, and the size of the data packet.

Proposed system mainly divides into two phases

1] EROB with Static Nodes

2] EROB with node mobility.

\subsection{EROB with Static Node}

To reduce the probability of collisions, and to simplify the collision prevention process, EROB uses three different channels. $\mathrm{CH}_{\mathrm{BIP}}$ and $\mathrm{CH}_{\text {DATA }}$ are dedicated to control packet (i.e., BIPs) and data packet transmissions, respectively. The usage of the third channel $\mathrm{CH}_{\mathrm{COL}}$ is to prevent the BIP Propagation Problem that will be explained shortly. Since different types of packets use different channels, collisions can occur only between the same type of packets, not between different types.

BIPs in EROB prevent data packet collisions as follows. Refer to Fig.1,BIPs are transmitted only along $\mathrm{CH}_{\mathrm{BIP}}$ and data packets are transmitted only along $\mathrm{CH}_{\text {DATA }}$. Suppose node $\mathrm{S}$ in Fig. 1 has a data packet to be one-hop broadcast. Then, prior to data transmission, S prepares and transmits a BIP with $\mathrm{TR}_{\mathrm{MAX}}\left(=\mathrm{TR}_{\mathrm{BIP}}\right)$ to inform the nodes in its transmission range with its intention of immediate data packet transmission.
Then, on receiving the BIP, if a node is currently not involved in any communication, then the node remains silent. Otherwise, i.e., if the node is currently involved in any other communication, it transmits a BIP to warn S not to transmit data packet, since S's transmission may cause collision at the node. On receiving either a BIP or a collision of BIPs, S refrains from transmitting data packet. Following two examples illustrate this case.

Case 1: Node in S's transmission range is currently involved in data packet transmission. Suppose there is a node, for example D in Fig.1(a), in S's transmission range that is involved in data packet transmission. Then, D prepares and transmits a BIP to warn S not to transmit data packet, since if $\mathrm{S}$ does, it would cause data packet collision at D. If there are two or more nodes that are involved in data transmission in S's transmission range, for example C and D in Fig.1(b), they all transmit BIPs to warn S. In this case, although $\mathrm{S}$ would hear a garbled message (i.e., garbled BIPs) that is not possible to decode correctly, $\mathrm{S}$ interprets the situation correctly and not to transmit data packet.

Case 2: Node in S's transmission range is currently involved in control packet transmission. Suppose there is more than one node, for example, $\mathrm{S}$ and $\mathrm{V}$ in Fig. 1 that When a node detects a BIP or a collision of BIPS in $\mathrm{CH}_{\mathrm{BIP}}$, it transmits a BIP only along $\mathrm{CH}_{\mathrm{COL}}$ indicating that the $\mathrm{BIP}$ is the response to the first BIP collision. For example, nodes $\mathrm{C}$ and $\mathrm{D}$ in Fig. 1(b) transmit a BIP only along $\mathrm{CH}_{\mathrm{COL}}$ when they detect a collision in $\mathrm{CH}_{\mathrm{BIP}}$ caused by two BIPs from $\mathrm{S}$ and $\mathrm{V}$. When $\mathrm{E}$ and $\mathrm{F}$ receive the garbled $\mathrm{BIP}$ along $\mathrm{CH}_{\mathrm{COL}}$, they know it is not the first BIP collision because it is along $\mathrm{CH}_{\mathrm{COL}}$, and do not transmit any BIP. Therefore, the BIP is dropped at C and D, and is not propagated to $G$ as well as not back to $C$ and $D$. Note that regardless of the reception along $\mathrm{CH}_{\mathrm{COL}}$, if a node detects a collision in $\mathrm{CH}_{\mathrm{BIP}}$, the node transmits a BIP along $\mathrm{CH}_{\mathrm{COL}}$.

EROB at each node executes the following phase.

Phase 1) Prior to 1-hop data packet broadcast, $S$ listens to the channels, CHBIP, CHDATA, and CHCOL for any on-going transmission. Then, the following two cases can occur.

Case 1) $S$ detects packet transmission in any of the three channels. This indicates that there is an on-going transmission in S's transmission range. Therefore, if S starts transmitting, it may cause collision. In this case, $\mathrm{S}$ waits a random amount of time, and then starts Phase 1 again.

Case 2) $\mathrm{S}$ does not hear any on-going packet transmission in any channel. In this case, $S$ will go to Phase 2.

Phase 2) $\mathrm{S}$ transmits a BIP packet along $\mathrm{CH}_{\mathrm{BIP}}$ with the maximum power $\mathrm{TR}_{\mathrm{MAX}}$ to inform its neighbor nodes with its intension of immediate transmission of data packet. During the BIP transmission, if S detects any on-going transmission except its transmission in any of three channels, then $S$ immediately stops transmitting the BIP and enters Phase 1 . Otherwise, after transmitting the BIP, S enters Phase 4. All other nodes including nodes in $\mathrm{TR}_{\mathrm{S}}$, MAX enter Phase 3.

Phase 3) A node in $\mathrm{TR}_{\mathrm{S}, \mathrm{BIP}}$ either receives a BIP or detects a collision along $\mathrm{CH}_{\mathrm{BIP}}$, depending on the number of BIP transmitting nodes within its $\mathrm{TR}_{\mathrm{MAX}}$. For example, suppose both $\mathrm{S}$ and $\mathrm{V}$ in Fig.1 transmit a BIP simultaneously. Then, node $\mathrm{F}$ hears the BIP from $\mathrm{S}$, but $\mathrm{D}$ detects a collision since it receives both BIPs from $S$ and $V$. Depending on the current status, a node, say $\mathrm{N}$ in $\mathrm{TR}_{\mathrm{S}, \mathrm{BIP}}, \mathrm{N} \neq \mathrm{S}$, falls into one of the following three cases. 
Case 1) $\mathrm{N}$ is currently transmitting a data packet. Note that $\mathrm{N}$ cannot be within $\mathrm{TR}_{\mathrm{s}, \mathrm{BIP}} / 2\left(=\mathrm{TR}_{\mathrm{S}, \mathrm{DATA}}\right)$, since if it is, $\mathrm{S}$ must have heard the data packet transmission from $\mathrm{N}$ in Phase 1 and could not enter Phase 2 .

Case 2) $\mathrm{N}$ is currently receiving a data packet. In this case, the location of $\mathrm{N}$ could be anywhere within $\mathrm{TR}_{\mathrm{S}, \mathrm{BIP}}\left(=\mathrm{TR}_{\mathrm{MAX}}\right)$.

To avoid a collision, node $\mathrm{N}$ transmits a BIP along $\mathrm{CH}_{\mathrm{COL}}$ with power level of $\mathrm{TR}_{\mathrm{MAX}} / 2$.

Case 3) $\mathrm{N}$ is not involved in any data packet transmission. In this case, $\mathrm{N}$ can be one of the following two sub cases.

Sub-case 1) V is receiving a single BIP. This implies that there is only one node ( $\mathrm{S}$ in this case) to be transmitting in V's transmission range. Since there is no possibility of collision, $\mathrm{V}$ keeps silent and does not do anything.

Sub-case 2) V is detecting a collision of BIPs in $\mathrm{CH}_{\mathrm{BIP}}$. This implies there is more than one node trying to transmit in V's transmission range, which may cause collision at $\mathrm{V}$. To prevent the collision, $\mathrm{V}$ transmits a $\mathrm{BIP}$ along $\mathrm{CH}_{\mathrm{COL}}$ with the power level of $\mathrm{TR}_{\mathrm{MAX}}$ to warn the nodes not to transmit.

Phase (4)After transmitting a BIP, S waits for response, if any. Then two cases can occur.

Case (1) S detects a BIP or a collision of BIPs in CHCOL. In this case, $\mathrm{S}$ stops and enters Phase 1.

Case (2) $S$ does not detect any signal in any channel. In this case, $\mathrm{S}$ can go ahead and transmits its data packet with $\mathrm{TR}_{\mathrm{MAX}} / 2$.

\subsection{EROB with Node Mobility}

if $\mathrm{S}$ and $\mathrm{V}$ are moving towards each other while transmitting data packets, an overlapped area will be formedeventually and may grow bigger, as shown in Fig.2(b). This will cause data packet collision at the nodes, for example $\mathrm{C}$ inthe overlapped area. Note that if there is a "enough distance" between $\mathrm{TR}_{\mathrm{S}, \mathrm{DATA}}$ and $\mathrm{TR}_{\mathrm{V}, \mathrm{DATA}}$ at the beginning of the data transmission, there would be no collision.This observation had led us to develop the following data collision avoidance scheme that guarantees the completion of one-hop broadcasting even in the presence of node mobility.

Suppose nodes S and V in Fig.2(a) just started transmitting data packets while moving towards each other with the speed of $\mathrm{SPEED}_{\mathrm{S}}$ and $\mathrm{SPEED}_{\mathrm{V}}$, respectively. Also, let $\mathrm{TIME}_{\mathrm{S}}$ and TIME $_{\mathrm{V}}$ be the data packet transmission time of $\mathrm{S}$ and $\mathrm{V}$, respectively. Note that this information is hidden from each other, because they even do not know the existence of each other. Our approach to avoid the possible collision is to give enough distance in advance between $\mathrm{TR}_{\mathrm{S}, \mathrm{DATA}}$ and $\mathrm{TR}_{\mathrm{V}, \mathrm{DATA}}$ so that they would not overlap during the data transmission. That is, if the distance between $\mathrm{S}$ and $\mathrm{V}$ is greater than SPEED $_{S} \times \mathrm{TIME}_{\mathrm{S}}+\mathrm{SPEED}_{\mathrm{V}} \times \mathrm{TIME}_{\mathrm{V}}$ when they start transmitting datapackets, there would be no collision. Since we may not be able to control the mobility of nodes, the only way to secure the distance to prevent the collision would be adjusting the transmission range. Therefore, if $\mathrm{S}$ reduces its transmission range $\mathrm{TR}_{\mathrm{S}, \mathrm{DATA}}$ by $\mathrm{D}_{\mathrm{S}}=\mathrm{SPEED}_{\mathrm{S}} \times \mathrm{TIME}_{\mathrm{S}}$, data packet collision could be avoided from S's side. However, since both $\mathrm{S}$ and $\mathrm{V}$ are moving towards each other in the worst case, $\mathrm{V}$ should also reduce its transmission range $\mathrm{TR}_{\mathrm{V}, \mathrm{DATA}}$ by $\mathrm{D}_{\mathrm{V}}=\mathrm{SPEED}_{\mathrm{V}} \times \mathrm{TIME}_{\mathrm{V}}$. Then, there will be no data packet collision, unless either $\mathrm{SPEED}_{\mathrm{S}}$ or $\mathrm{SPEED}_{\mathrm{V}}$ increases.

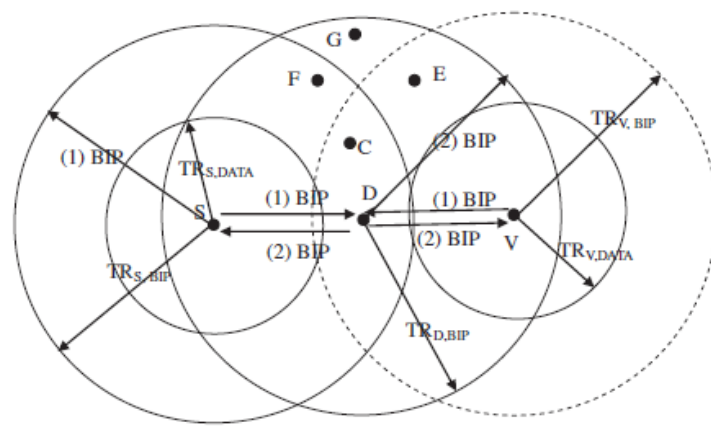

(a)

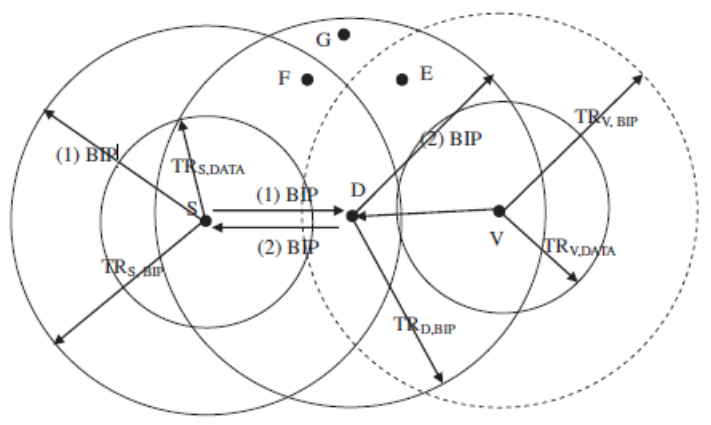

(b)

Figure 1: Examples of how BIP used to prevent collisions. (a)only one node. (b) more than one node

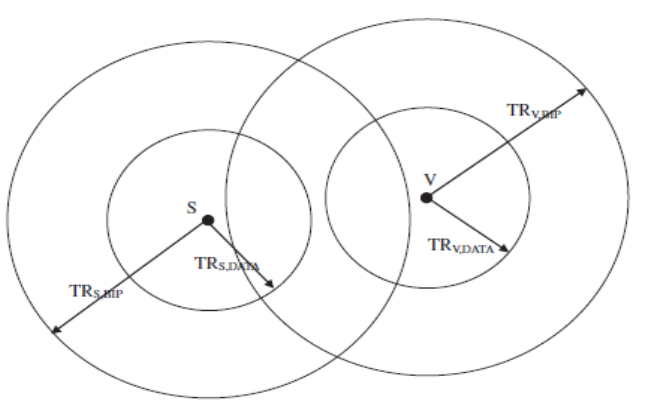

(a)

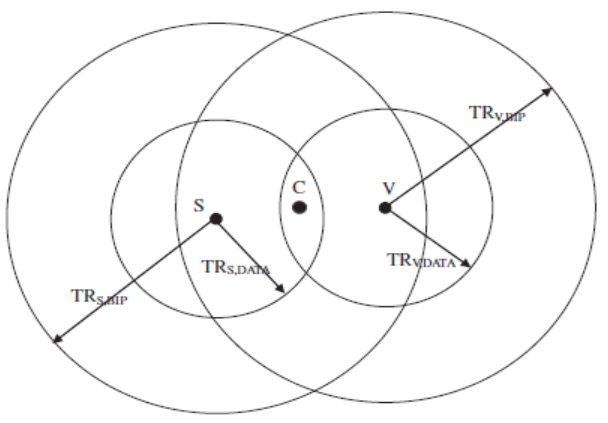

(b)

Figure.2.Nodes $\mathrm{S}$ and $\mathrm{V}$ are transmitting data packets while they are moving towards each other (a)when nodes $S$ and $V$ start transmitting data packets, no collisions b)sometimes later, there will be a collision at node $\mathrm{C}$ that is in $T R_{\text {S,DATA }} \cap T R_{V, \text { DATA }}$. 


\section{RESULTS AND DISCUSSIONS}

The proposed protocol is implemented in Network Simulator (NS2) software. The parameters used for comparison between CSMA and EROB protocols are packet delivery ratio, control overhead, normalized routing overhead, delay, throughput, delay, jitter, dropping ratio. All these parameters are analyzed with network simulator and their performances are presented in Figure 2 to Figure 7 and the analysis of all these figures is summarized in Table 2. Simulation parameters used are listed in Table 1 for quick reference.

Table 1:Simulation Parameters

\begin{tabular}{|c|c|}
\hline Parameters & Value \\
\hline Source Type & MAC \\
\hline No. of Nodes & $50,60,70,80,90,100$ \\
\hline Simulation Time & $200 \mathrm{sec}$ \\
\hline Traffic Type & CBR \\
\hline Transmission Range & $1000 * 1000$ \\
\hline Simulation Area & $20 \mathrm{~m} / \mathrm{s}$ \\
\hline Node Speed & $2000 \mathrm{bytes}$ \\
\hline Packet Size & 00ms \\
\hline Pause Time & CSMA,EROB \\
\hline Routing Protocol & \\
\hline
\end{tabular}

Following points are noted from Figure 3 to Figure 8 .

- When speed of node increases PDR of CSMA is decreases and PDR of EROB increases as shown in Figure3.

- When speed of node increases control overhead of CSMA decreases while control overhead of EROB is increases as shown in figure 4. This ratio is calculated by comparing the total number of routing packets transmitted during the simulation time to the number of data packets delivered.

- Simulated values of delay represent reliability of routing protocol in the network. As speed increases delay of CSMA increases while delay of EROB decreases.

- Figure 5 shows when speed of nodes increases throughput of CSMA increases while throughput of EROB decreases.

- Jitter is reduced with time. Jitter is employed to avoid collisions caused by simultaneous transmission by adjacent nodes over the same channel. As the number of packets increased over the same channel, jitter is increased which leads to the loss of data.(Figure 7)

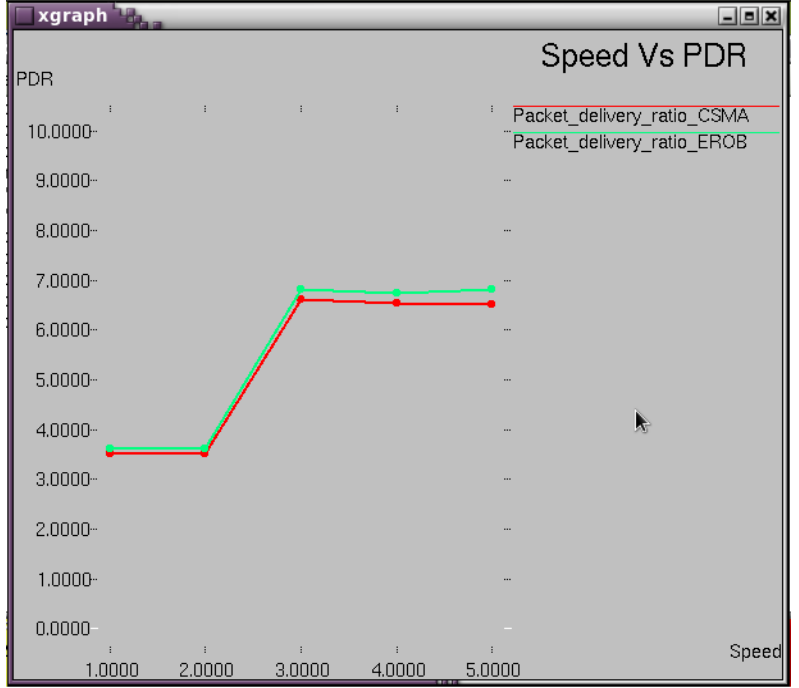

Figure 3:Speed Vs Packet delivery ratio(PDR)

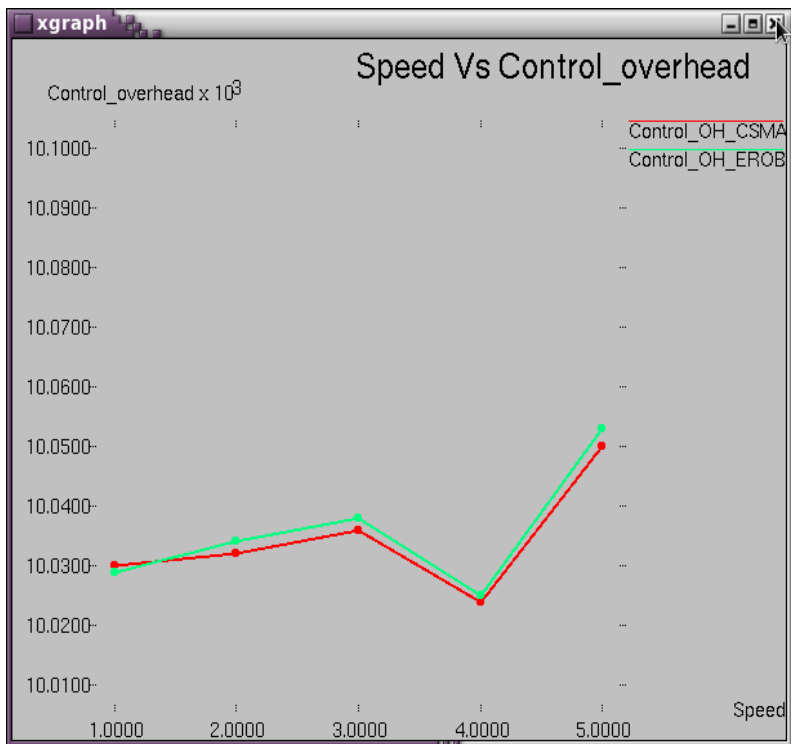

Figure 4:Speed Vs Control Overhead

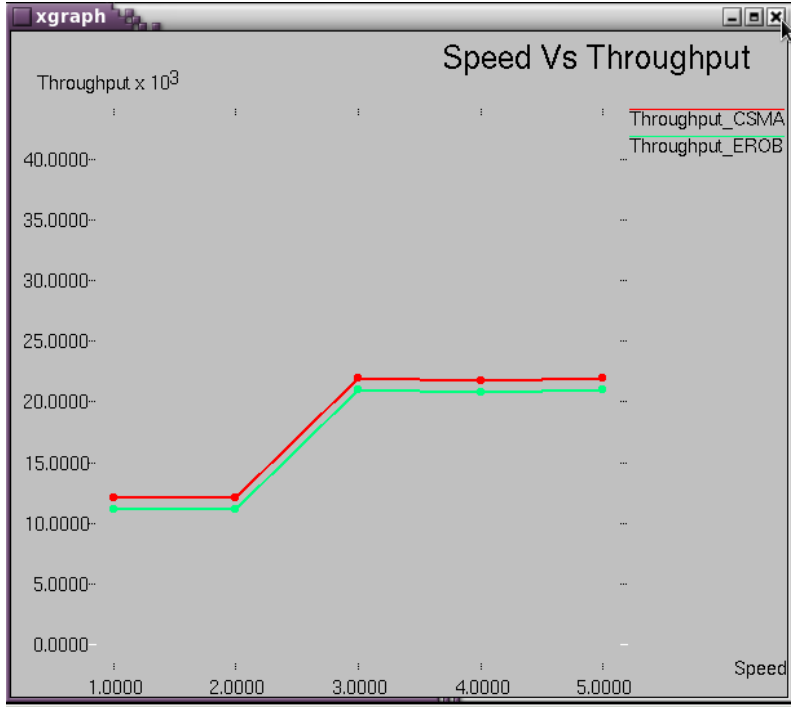

Figure 5: Speed Vs Throughput 


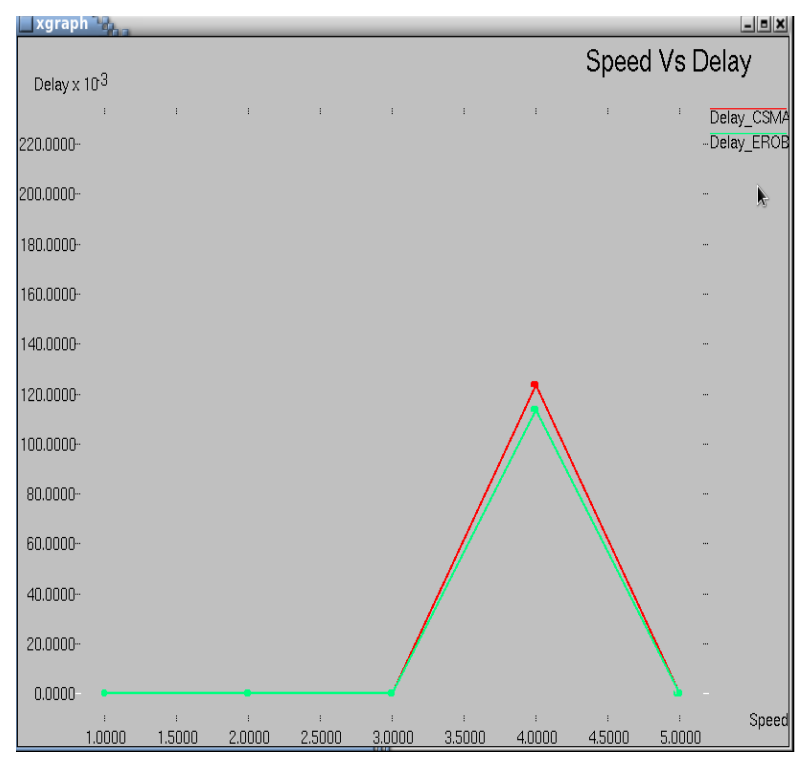

Figure 6:Speed Vs Delay

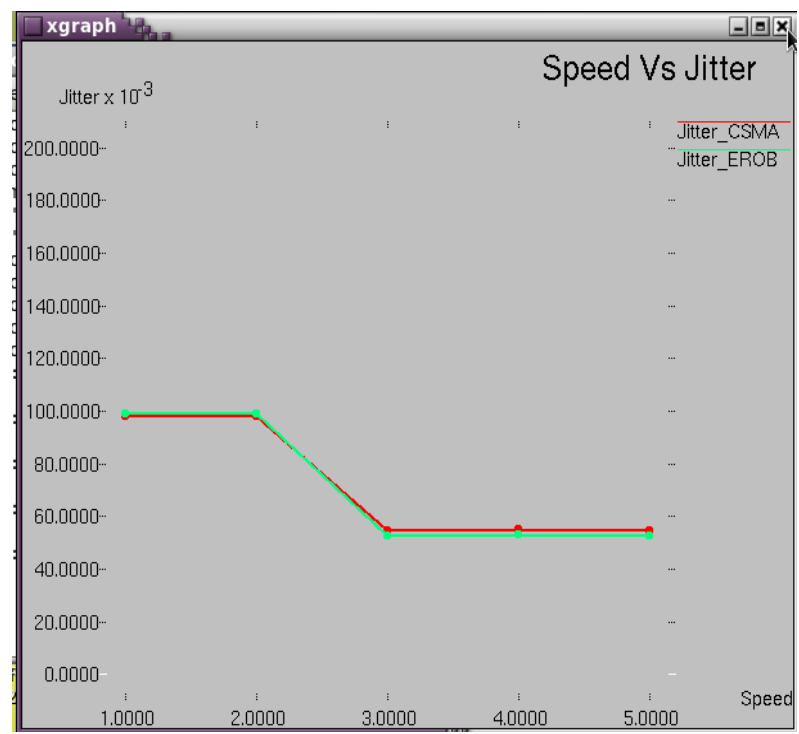

Figure 7:Speed Vs Jitter

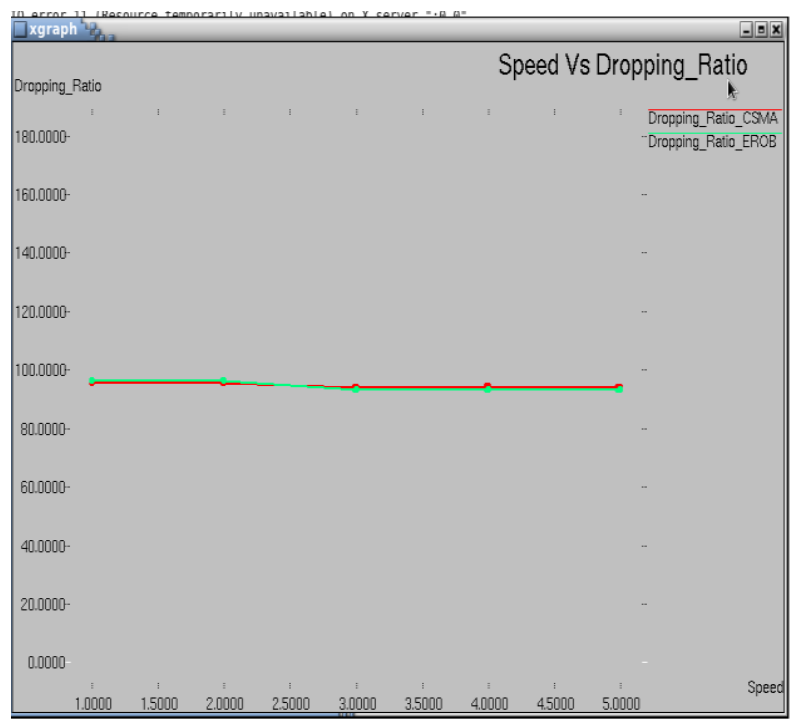

Figure 8: Speed Vs Dropping ratio
Following points are noted from Figure 9 to Figure 14.

The performance of protocol is compared along with presence of traffic nodes 50,60, 80,90 and 100 and with delay, throughput ,pdr etc

- When number of nodes increases PDR of CSMA is increases and PDR of EROB decreases as shown in Figure9.

- When number of nodes increases control overhead of CSMA decreases while control overhead of EROB is increases as shown in figure 4. This ratio is calculated by comparing the total number of routing packets transmitted during the simulation time to the number of data packets delivered.

- Simulated values of delay represent reliability of routing protocol in the network. As number of nodes increases delay of CSMA increases while delay of EROB decreases.

- Figure 5 shows when number of nodes increases throughput of CSMA increases while throughput of EROB decreases.

- Jitter is reduced with time. Jitter is employed to avoid collisions caused by simultaneous transmission by adjacent nodes over the same channel. As the number of packets increased over the same channel, jitter is increased which leads to the loss of data.

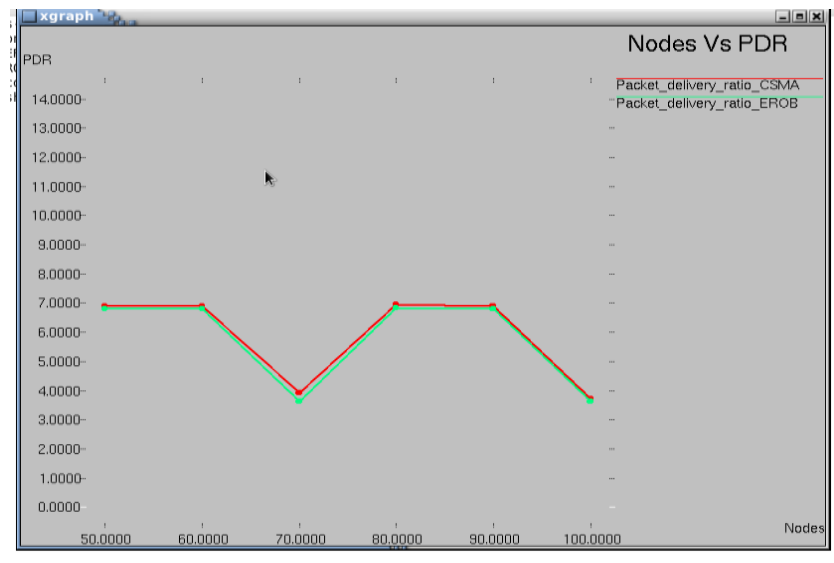

Figure 9:Nodes Vs PDR

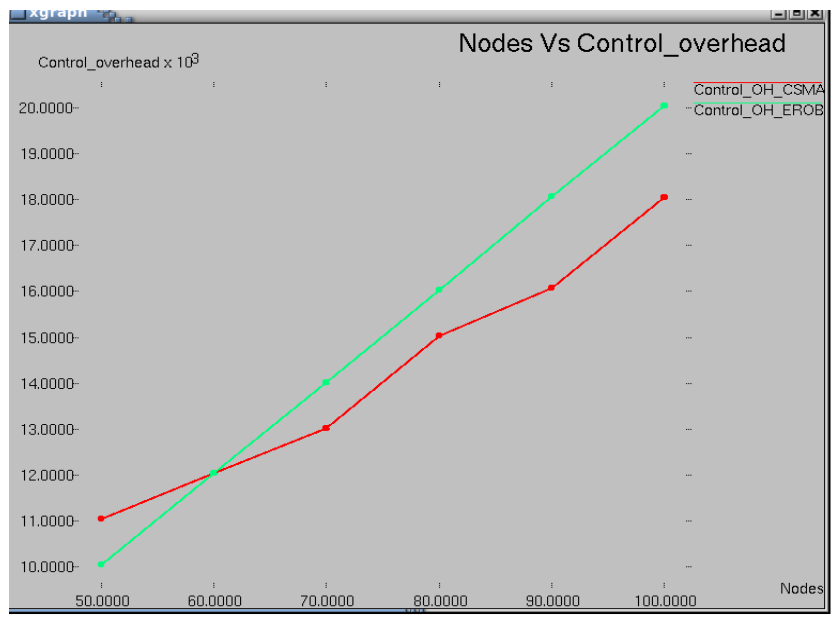

Figure 10:Nodes Vs Control Overhead 


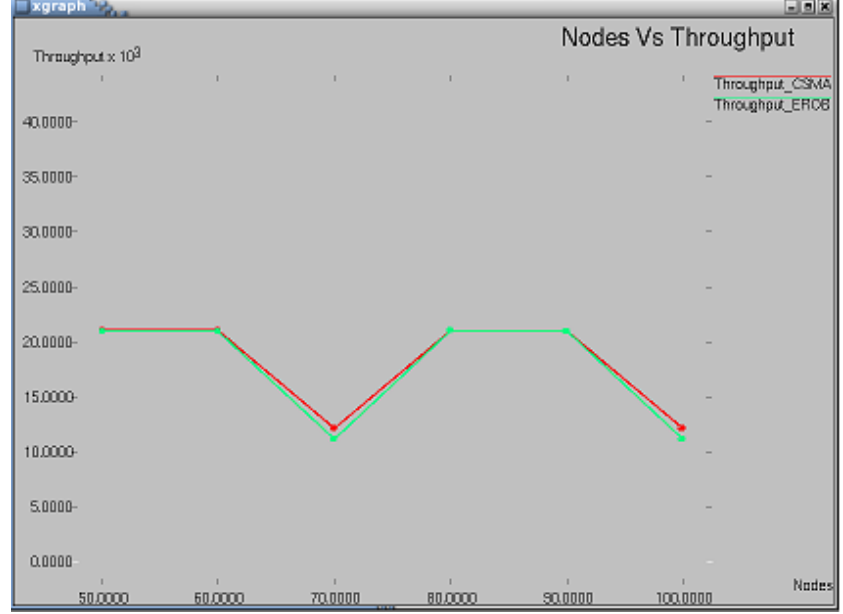

Figure 11:Nodes Vs Throughput

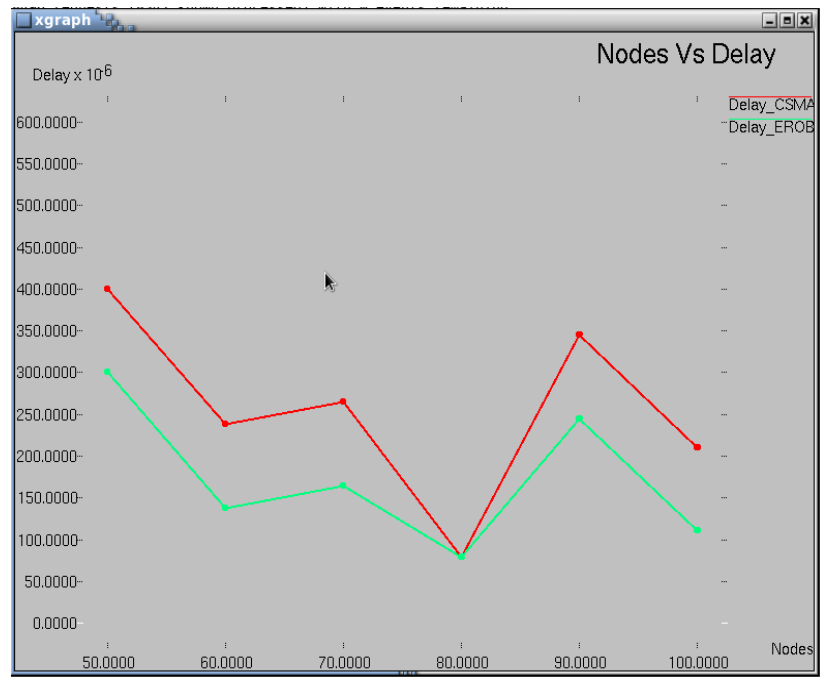

Figure 12 :Nodes Vs Delay

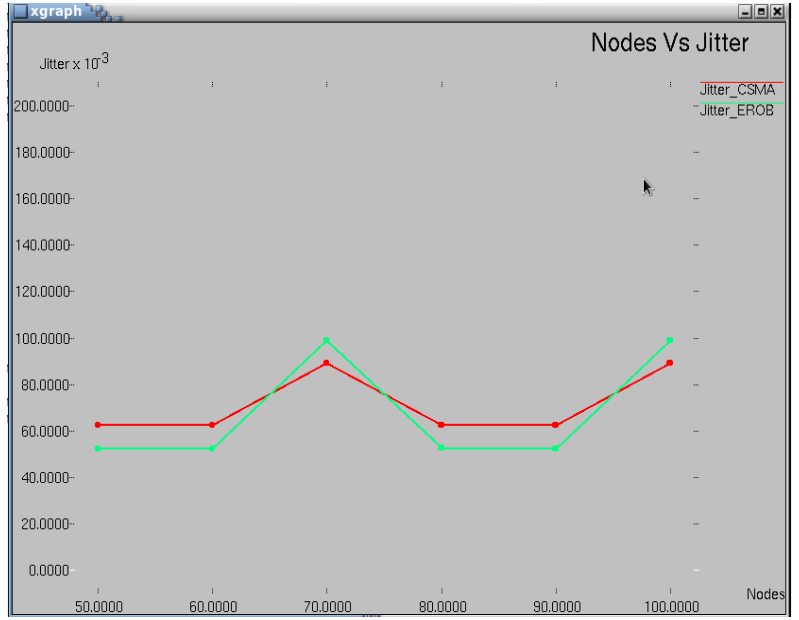

Figure 13: Nodes Vs Jitter

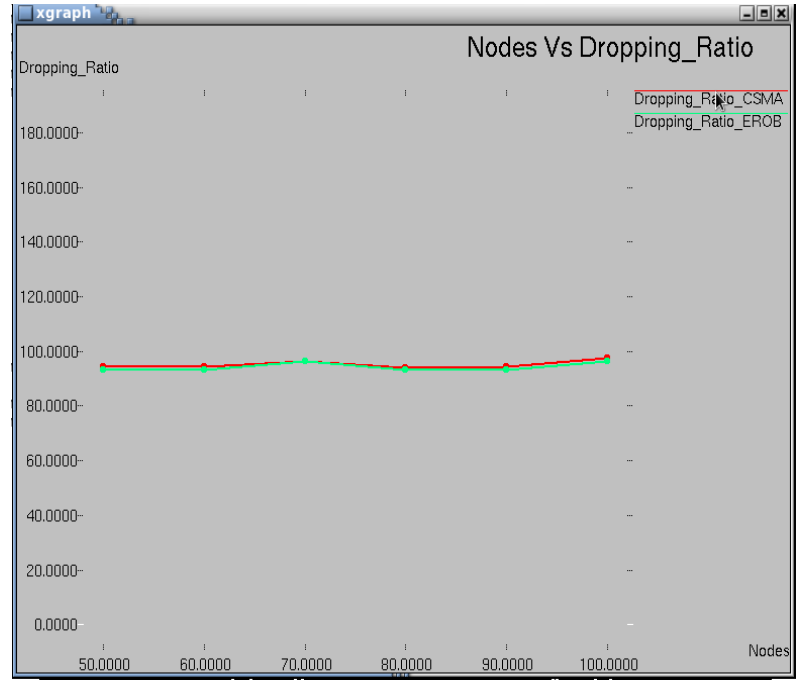

Figure 14 :Nodes Vs Dropping Ratio

Table 2:Performance analysis of different parameters

\begin{tabular}{|l|l|l|l|l|l|l|}
\hline Parameters & \multicolumn{1}{|c|}{ PDR } & \multicolumn{1}{c|}{$\begin{array}{c}\text { Control } \\
\text { overhead }\end{array}$} & \multicolumn{1}{|c|}{ Throughput } & \multicolumn{1}{|c|}{ Delay } & Jitter & \multicolumn{1}{|c|}{ Dropping Ratio } \\
\hline CSMA & Decreases & Decreases & Increases & Increases & Increases & Increases \\
\hline Static EROB & Increases & Increases & Decreases & Decreases & Decreases & Decreases \\
\hline Dynamic EROB & Increases & Increases & Decreases & Decreases & Constant & Decreases \\
\hline
\end{tabular}

\section{CONCLUSION}

One-hop broadcast is a fundamental communication primitive in any networks, especially in wireless networks where every transmission is a1-hop broadcast by nature.

We have presented an algorithm, called EROB, that guarantees the completion of 1-hop broadcast in mobile adhoc networks.

A node implementing EROB uses different transmission power levels depending on its current status and the type of the communication it is currently involved. Broadcasting nodes transmit BIPs with transmission range of $\mathrm{TR}_{\mathrm{MAX}}$ (maximum power level) and data packets and receiving nodes transmit BIPs, if necessary, with power level that is enough to reach destination. Because that the diameter of transmission range in EROB is about the half of possible maximum size, this scheme provides many advantages including

- $\quad$ power saving due to smaller transmission range

- less collision with less number of nodes in transmission ranges, and

- better network throughput via more simultaneous transmissions.

This paper presents the results and performance analysis of two protocols i.e CSMA and EROB .Table 2 and figures 
presents the effective performance of proposed system over existing system.

\section{REFERENCES}

[1] D. Allen, - Hidden Terminal Problems in Wireless LAN's,\| IEEE 802.11 Working Group paper 802.11/93$\mathrm{xx}$.

[2] V. Bharghavan et al., -MACAW: A Media Access Protocol for Wireless LAN's,\|Proc. ACM SIGCOMM, 1994.

[3] IEEE 802.11 Working Group, - Wireless LAN Medium Access Control (MAC) and Physical Layer (PHY) Specifications,॥ 1997.

[4] J. Deng and Z. Haas, -Dual Busy Tone Multiple Access (DBTMA): A New Medium Access Control for Packet Radio Networks,\|Proc. of IEEE ICUPC, 1998.

[5] Z. Tang and J.J Garcia-Luna-Aceves, -Hop-Reservation Multiple Access (HRMA) for Ad-Hoc Networks, Proc. of IEEE INFOCOM, 1999.

[6] Tzamaloukas and J.J. Garcia-Luna-Aceves, -A Receiver-Initiated Collision-Avoidance Protocol for Multi-Channel Networks,\|Proc. of IEEE INFOCOM, 2001 .

[7] M. Mohsin, D. Cavin, Y. Sasson, R. Prakash, and A. Schiper, - Reliable Broadcast in Wireless Mobile Ad Hoc Networks, Proc. of 39th Hawaii International Conference on System Science, 2006.

[8] S. Park and R. Palasdeokar, -Reliable One-Hop Broadcasting (ROB) in Mobile Ad Hoc Networks, $\|$ 2nd ACM International Workshop on Performance Evaluation of Wireless Ad Hoc, Sensor, and Ubiquitous Networks (PE-WASUN '05), pp. 234-237, 2005.
[9] J. Lembke, Z. Ryne, H. Li, S. Park, -Collision Avoidance in One-Hop Broadcasting for Mobile Ad-hoc Networks,\| IASTED International Conference on Communication, Internet, and Information Technology, pp. 308-313, 2005.

[10] S. Park, R. Anderson, Guaranteed one-hop broadcasting in mobile adhoc networks. in: The 2008 International Conference on Parallel and Distributed Processing Techniques and Applications (PDPTA'08), July 14-17, 2008.

[11] Jie Wu, Fei Dai, Broadcasting in ad hoc networks based on selfpruning, in: INFOCOM, 2003.

[12] Y. Li, M.T. Thai, F. Wang, C.-W. Yi, P.-J. Wan, D.-Z $\mathrm{Du}$, On greedy construction of connected dominating sets in wireless networks, Wireless Communications and Mobile Computing (WCMC) 5 (8) (2005) 927-932.

[13] P.-J. Wan, K.M. Alzoubi， O. Frieder, Distributed construction on connected dominating set in wireless ad hoc networks, Mobile Networks and Applications 9 (2) (2004) 141-149.

[14] J. Haas, A new routing protocol for the reconfigurable wireless networks, in: Proc. of IEEE 6th International Conference on Universal Personal Communications, vol. 97, 1997, pp. 562-566.

[15] H. Lim, C. Kim, Multicast tree construction and flooding in wireless ad hoc networks, in: Proceedings of the Third ACM International Workshop on Modeling, Analysis and Simulation of Wireless and Mobile Systems (MSWiM), August 2000.

[16] P. Karn, MACA - a new channel access method for packet radio, in: ARRL/CRRL Amateur Radio 9th Computer Networking Conference, September 22, 1990. 